\title{
The Problem of Noncharacteristic Quasimolecular $X$ Rays in Heavy Ion Collision
}

\author{
T. MOROVIĆ AND B. FRICKE \\ Gesamthochschule Kassel, D-3500 Kassel, Germany
}

A. ROSÉN

Chalmers University of Technology, S-40220 Göteborg, Sweden

\begin{abstract}
AND
D. E. ELLIS

Northwestern University, Evanston, Illinois 60201, and Scheikundig Laboratorium der Vrije Universiteit, Amsterdam, Netherlands
\end{abstract}

\begin{abstract}
Quasi-molecular $\mathrm{X}$ rays observed in heavy ion collisions are interpreted within a relativistic calculation of correlation diagrams using the Dirac-Slater model. A semiquantitative description of noncharacteristic $M$ $\mathrm{X}$ rays is given for the system $\mathrm{Au}-\mathrm{I}$.
\end{abstract}

During heavy ion-atom collisions noncharacteristic $\mathrm{X}$ rays in various colliding systems have been observed. The data available can be divided into three different groups: the quasimolecular $K \mathrm{X}$ rays observed in the systems with low and medium $Z$ [1], a few observations of quasimolecular $L \mathrm{X}$ rays in medium $Z$ systems [2], and the observations connected with quasimolecular $M \mathrm{X}$ rays in heavy systems [3]. It seems clear that a correct interpretation of this phenomenon can only be given by a full self-consistent $d y-$ namical description of the collision, which at present is out of practical range. Nevertheless, one can expect that the interpretation of the structureless $K \mathrm{X}$-ray spectra through the static relativistic correlation diagrams which hold only one electron and are easy to calculate represents a good approximation, because the screening effects are small and only a few levels and thus a few level crossings are involved [4].

In contrast to the $K$ and $L \mathrm{X}$-ray spectra a significant structure in $M \mathrm{X}$-ray spectra has been found [3], the best known example being the Au-I system. As discussed in an earlier paper [5], the most important difference in the correlation diagrams for the $M$ quasimolecular radiation compared with correlation diagrams for the $K$ or $L X$-ray spectra is a very flat and wide minimum of the second $\pi$ level which ends in the $3 d_{3 / 2}$ level of the combined system. Such a flat minimum at the internuclear distance of about 0.1 a.u. may then be the reason for the quasimolecular peak in observed spectra. This is only a qualitative interpretation which has to be clarified by better calculations. 


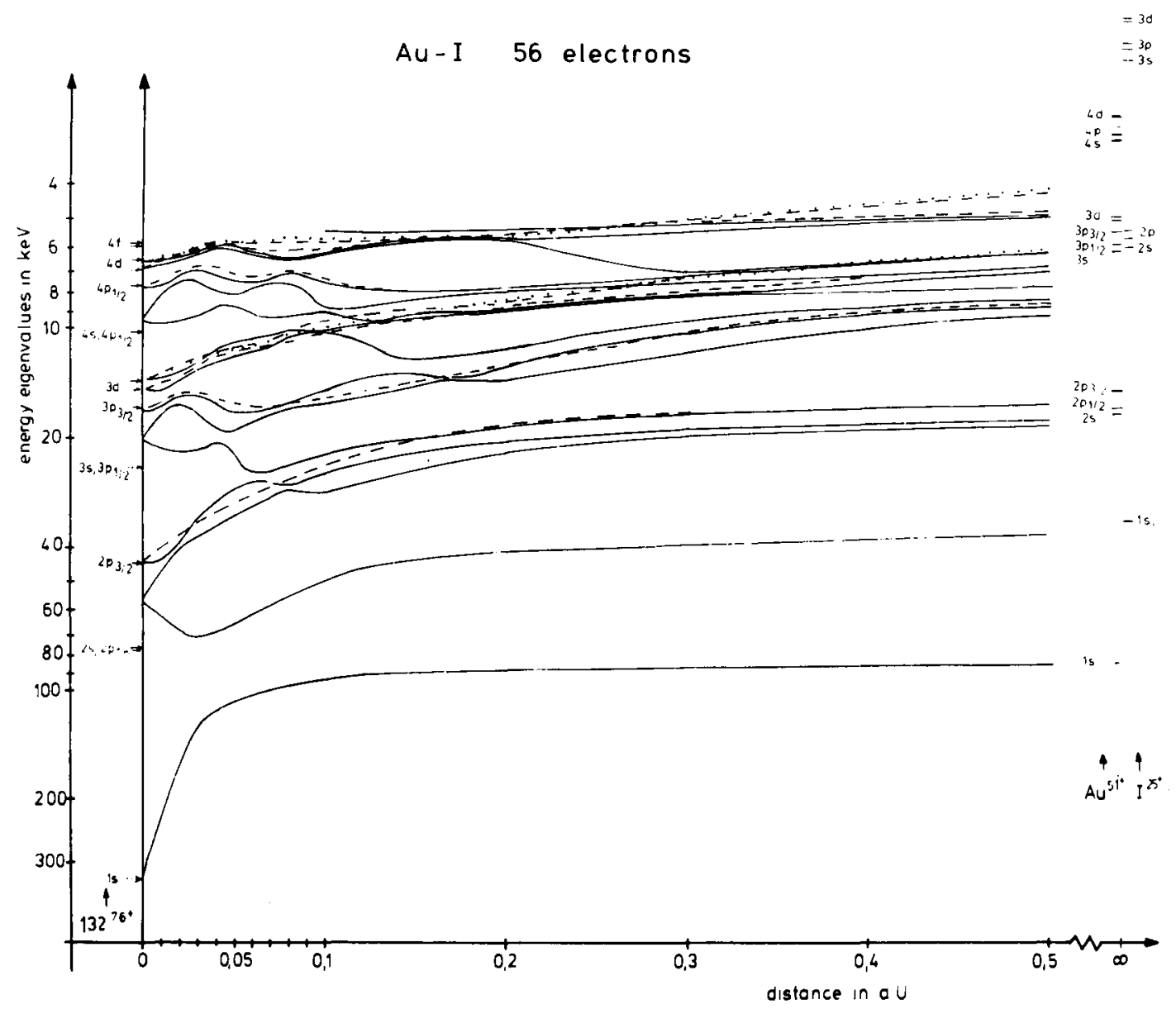

Figure 1. Self-consistent Dirac-Slater one-electron energies for the Au-I system versus internuclear separation $R$.

Recently an ab initio self-consistent field relativistic Dirac-Slater molecular program for obtaining molecular energy levels and wave functions has been developed. Extended calculations for systems like $\mathrm{XeF}_{2}, \mathrm{InI}, \mathrm{UO}_{2}{ }^{2+}[6,7]$ showed its usefulness. In the Dirac-Slater Hamiltonian

$$
h=c \alpha p+\beta c^{2}+1 V(r)
$$

the molecular potential is represented as a sum of the Coulomb and exchange terms, the latter being proportional to $[\rho(r)]^{1 / 3}$ as usual in the local density approximation, where $\rho(\boldsymbol{r})$ is taken to be the total charge density.

The eigenfunctions of the relativistic molecular Hamiltonian are constructed as linear combinations of atomic basis functions which are taken as the numerical Dirac-Slater eigenfunctions for atoms/ions located at given sites. For obtaining the energy eigenvalues and eigenfunctions of the molecular system the discrete variational procedure [8] was applied. The resulting matrix secular equation has been reduced by taking linear combinations of the basis functions which transform according to irreducible representation of the molecular double point group [9]. In order to obtain self-consistency, after each iteration a Mullikan population analysis has been performed and the resulting occupation 


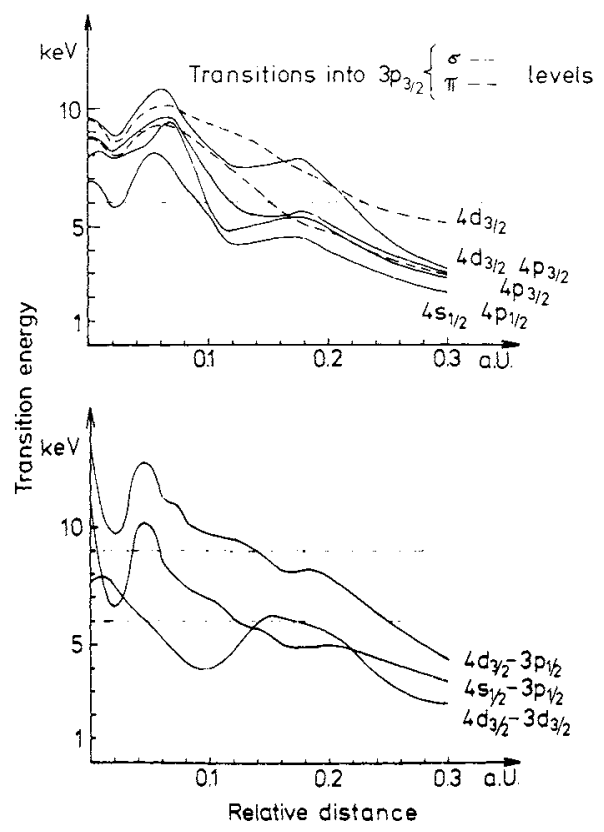

Figure 2. Transition energies $\epsilon_{j}-\epsilon_{i}$ for $M$ X-ray transitions. Notation refers to united atom levels.

numbers were then used to construct a new molecular potential for the next iteration.

We have used this program to explore the collision process of the well-known Au-I system where quasimolecular $M \mathrm{X}$ rays have been observed [3]. The resulting correlation diagram of this system, taking into account 56 electrons, is shown in Figure 1. Since there are no measurements of the actual degree of ionization of the system, we have chosen the number of 56 electrons as a convenient reasonable assumption. With this number of electrons all levels up to the main quantum number $n=4$ of the combined system are occupied. One knows from atomic calculations that the inclusion of more electrons has no significant effect on the transition energies between levels already occupied. In the actual calculations we have included a large number of $s$-, $p$ - and $d$-type basis functions, but no $f$ states have been used, although their inclusion is desirable in order to describe $p$-shell polarization. It will be clear that for sufficiently small internuclear distances $R$ the elements of the two-center expansion basis set become nearly linearly dependent, and precautions must be taken to avoid difficulties. In the present work this problem appeared for $R \leqslant 0.05$ a.u. The most strongly affected levels correspond to $j=1 / 2$ eigenstates of the united atom, which leads to too small binding energies. The degenerate basis functions have been easily identified due to Mullikan population analysis. For very small $R$ this problem can be solved by dropping one basis set and performing a one-center expansion. This expansion converges well and provides a satisfactory starting point for extension by atom in well procedures $[10]$ if needed.

From the correlation diagram shown in Figure 1 we have extracted the $M$ transition energies as a function of the internuclear distance $R$. Figure 2 shows interesting and unexpected results. In the relevant energetical range between 6 and $9 \mathrm{keV}$, where the experimental noncharacteristic X-ray peak appears, we find remarkable structures in the radial behavior of the transition energies. But most important of all is the behavior of the transition energy curve which remains nearly constant at $7.5 \mathrm{keV}$ between $R=$ 
0.12 a.u. and $R=0.19$ a.u. This result indicates that the experimental quasimolecular peak may be produced mainly by this constant behavior of one of the transition energies plus a superposition of the relatively complex behavior of all other quasimolecular transitions in this energetical range. We still do not know at the present stage of the experimental and theoretical evaluation the number of holes created in the various levels during the collision as well as the total ionicity.

In order to describe the noncharacteristic X-ray structure in a more quantitative way, we have constructed the joint density of states as a function of the internuclear distance using the data of Figure 2. Then the life time broadening, which is a function of the projectile velocity, has to be folded in. This has to be weighted with the transition probability for every transition, and finally this has to be integrated over $R$ and all impact parameters for given projectile energy. The preliminary results are very encouraging, but we are still assuming the isotropy of the radiation, neglecting every dynamical effect like change in occupation probabilities during the collision. All these omissions will not affect the position of the noncharacteristic X-ray peak very much, provided that the whole system behaves in a relatively adiabatic manner.

\section{Acknowledgment}

This work was supported in part by GSI, Darmstadt, Germany.

\section{Bibliography}

[1] J. S. Greenberg, C. K. Davis, and P. Vincent, Phys. Rev. Lett. 33, 473 (1974).

[2] W. Wölfli, C. Stoller, G. Bonani, M. Suter, and M. Stöckli, Proc. 9th Int. Conf. on the Physics of Electronic and Atomic Collisions, (Seattle, Wash., July 1975).

[3] F. W. Saris, W. F. van der Weg, H. Tawara, and R. Laubert, Phys. Rev. Lett. 28, 717 (1972); P. H. Mokler, H. J. Stein and P. Armbruster, Phys. Rev. Lett. 29, 827 (1972).

[4] B. Müller and W. Greiner, Z. Naturforsch. 31a, 1 (1976).

[5] B. Fricke, K. Rashid, P. Bertoncini, and A. C. Wahl, Phys. Lett. 34, 243 (1975).

[6] A. Rosén and D. E. Ellis, J. Chem. Phys. 62, 3039 (1975).

[7] D. E. Ellis, A. Rosén, and P. F. Walch, Int. J. Quant. Chem. 9, 351 (1975).

[8] D. E. Ellis and G. S. Painter, Phys. Rev. B2, 2887 (1970); E. J. Baerends, D. E. Ellis, and P. Ros, Chem. Phys. 2, 41 (1973).

[9] Y. Onodera and M. Okazaki, J. Phys. Soc. Japan 21, 2400 (1966).

[10] F. W. Averill and D. E. Ellis, J. Chem. Phys. 59, 6412 (1973).

Received March 22, 1976 